\title{
A Gibbs Sampler for a Hierarchical Dirichlet Process Mixture Model
}

Mark Andrews

April 11, 2019

$\mathrm{T}$ HE hierarchical Dirichlet process mixture model (HDPMM), when its mixture components are categorical distributions, is a probabilistic model of multinomial data. It was first described as part of a more general description of Hierarchical Dirichlet Process (HDP) models by Teh, Jordan, Beal, and Blei $(2004,2006)$, and is the Bayesian nonparametric generalization of the Latent Dirichlet Allocation (LDA) model of Blei, Ng, and Jordan (2003).

The aim of this note is to describe in detail a Gibbs sampler for the HDPMM when used with multinomial data. This Gibbs sampler is based on of what was described in Teh et al. $(2004,2006)$ for general HDP models. However, as they did not deal in detail with HDP mixture models for multinomial data, important details of the sampler required for this particular case were not described. On the other hand, Newman, Asuncion, Smyth, and Welling (2009) do deal explicitly with the case of the HDPMM for multinomial data, and the Gibbs sampler that we describe here is almost identical to theirs. However, for some hyper-parameters, Newman et al. (2009) either make simplifying assumptions or assume that their values are known, which are assumptions that we do not make here. As such, the sampler we describe here is an extension, though minor, of that described in Newman et al. (2009).

\section{The probabilistic model}

One of the most straightforward applications of the multinomial data HDPMM is as a bag-of-words probabilistic language model, and in what follows we'll describe it with this application in mind. However, modulo some possible changes in notation, this will in fact constitute a general description of the HDPMM for multinomial data.

In general, according to a bag-of-words language model, a corpus of natural language is a set of $J$ documents or texts

$$
w_{1}, w_{2} \ldots w_{j} \ldots w_{\mathrm{J}}
$$

where text $j$, i.e., $w_{j}$, is a set of $n_{j}$ words from a finite vocabulary of $V$ word types. For simplicity, this vocabulary can be represented as the $V$ integers $\{1,2 \ldots V\}$. From this, we have each $w_{j}$ defined as

$$
w_{j}=w_{j 1}, w_{j 2} \ldots w_{j i} \ldots w_{j n_{j}}
$$

with each $w_{j i} \in\{1 \ldots V\}$. The bag-of-words assumption is that, for each text, $w_{j 1}, w_{j 2} \ldots w_{j i} \ldots w_{j n_{j}}$ are exchangeable random variables, i.e. their joint probability distribution is invariant to any permutation of the indices. By this assumption therefore, as the name implies, each text is modelled as an unordered set, or bag, of words.

As a generative model of this language corpus, the HDPMM treats each observed word $w_{j i}$ as a sample from one of an underlying set of text or discourse topics:

$$
\phi=\phi_{1}, \phi_{2} \ldots \phi_{k} \ldots,
$$


where each $\phi_{k}$ is a probability distribution over $\{1 \ldots V\}$. The identity of the particular topic distribution from which $w_{j i}$ is drawn is determined by the value of a discrete latent variable $x_{j i} \in\{1,2 \ldots k \ldots\}$ that corresponds to $w_{j i}$. As such, for each $w_{j i}$, we model it as

$$
w_{j i} \mid x_{j i}, \phi \sim \operatorname{dcat}\left(\phi_{x_{j i}}\right) .
$$

To be clear, the HDPMM assumes that there are an unlimited number of topic distributions from which the observed data are drawn, and so each $x_{j i}$ can take on infinitely many discrete values. The probability distribution over the infinitely possible values of each $x_{j i}$ is given by an infinite length categorical distribution $\pi_{j}$, i.e., $\pi_{j}=\pi_{j 1}, \pi_{j 2} \ldots \pi_{j k} \ldots$, where $0 \leqslant \pi_{j k} \leqslant 1$ and $\sum_{k=1}^{\infty} \pi_{j k}=1$, that is specific to text $j$. In other words,

$$
x_{j i} \mid \pi_{j} \sim \operatorname{dcat}\left(\pi_{j}\right) .
$$

Each $\pi_{j}$ is assumed to be drawn from a Dirichlet process prior whose base distribution, $m$, is a categorical distribution over the positive integers and whose scalar concentration parameter is a:

$$
\pi_{\mathfrak{j}} \mid a, m \sim \operatorname{ddp}(a, m) .
$$

The $m$ base distribution is assumed to be drawn from a stick breaking distribution with a parameter $\gamma$ :

$$
\mathrm{m} \mid \gamma \sim \operatorname{dstick}(\gamma) .
$$

The prior distributions of the Dirichlet process concentration parameter a and the stick-breaking parameter $\gamma$ are Gamma distributions, both with shape and scale parameters equal to 1 . For the topic distributions, $\phi_{1}, \phi_{2} \ldots \phi_{k} \ldots$, we can assume they are independently and identically drawn from a Dirichlet distribution with a length $V$ location parameter $\psi$ and concentration parameter b. In turn, $\psi$ is drawn from a symmetric Dirichlet distribution with concentration parameter $c$. Finally, both $b$ and $c$, like $a$ and $\gamma$, can be given Gamma priors, again with shape and scale distributions equal to 1 .

\section{Sampling each latent variable $\mathrm{x}_{\mathrm{ji}}$}

The posterior probability that $x_{j i}$ takes the value $k$, for any $k \in 1,2 \ldots$, is

$$
\begin{aligned}
\mathrm{P}\left(x_{j i}=k \mid w_{j i}\right. & \left.=v, x_{\neg j i}, w_{\neg j i}, b, \psi, a, m\right), \\
& \propto P\left(w_{j i}=v \mid x_{j i}=k, w_{\neg j i}, x_{\neg j i}, b, \psi\right) P\left(x_{j i}=k \mid x_{\neg j i}, a, m\right),
\end{aligned}
$$

where $x_{-j i}$ denotes all latent variables excluding $x_{j i}$, with an analogous meaning for $w_{\neg j i}$. Here, the likelihood term is

$$
\mathrm{P}\left(w_{j i}=v \mid x_{j i}=k, x_{\neg j i}, w_{\neg j i}, b, \psi\right)=\int \mathrm{P}\left(w_{j i}=v \mid \phi_{k}\right) \mathrm{P}\left(\phi_{k} \mid x_{\neg j i}, w_{\neg j i}, b, \psi\right) d \phi_{k} .
$$

This is the expected value of $\phi_{k v}$ according the Dirichlet posterior

$$
\mathrm{P}\left(\phi_{\mathrm{k}} \mid \mathrm{x}_{\neg j \mathrm{j}}, w_{\neg j \mathrm{j}}, \mathrm{b}, \psi\right)=\frac{\Gamma\left(\mathrm{S}_{\mathrm{k}}^{\neg \mathrm{ji}}+\mathrm{b}\right)}{\prod_{v=1}^{V} \Gamma\left(\mathrm{S}_{\mathrm{k} v}^{\neg j \mathrm{j}}+\mathrm{b} \psi_{v}\right)} \prod_{v=1}^{\mathrm{V}} \phi_{\mathrm{kv}}^{\mathrm{S}_{\mathrm{k} v}^{-j i}+\mathrm{b} \psi_{v}-1},
$$

where $S_{k v}^{\neg i} \triangleq \sum_{j / i / \neq j i} \mathbb{I}\left(w_{j / i}=v, x_{j / i}=k\right)$ and $S_{k}^{\neg j i}=\sum_{v=1}^{V} S_{k v}^{\neg j i}$. As such,

$$
\mathrm{P}\left(w_{j i}=v \mid x_{j i}=k, x_{\neg j i}, w_{\neg j l i}, b, \psi\right)=\frac{S_{k v}^{\neg j i}+b \psi_{v}}{S_{k \cdot}^{\neg j i}+b} .
$$


The prior term, on the other hand, is

$$
\mathrm{P}\left(x_{j i}=k \mid x_{\neg j i}, a, m\right)=\int P\left(x_{j i}=k \mid \pi_{j}\right) P\left(\pi_{j} \mid x_{\neg j i}, a, m\right) d \pi_{j},
$$

and this the expected value of $\pi_{j k}$ according to

$$
\mathrm{P}\left(\pi_{\mathfrak{j}} \mid x_{\neg j i}, \mathrm{a}, \mathrm{m}\right) \propto \mathrm{P}\left(x_{\neg j \mathrm{j}} \mid \pi_{\mathfrak{j}}\right) \mathrm{P}\left(\pi_{\mathfrak{j}} \mid \mathrm{a}, \mathrm{m}\right) .
$$

As $\mathrm{P}\left(\pi_{\mathrm{j}} \mid \mathrm{a}, \mathrm{m}\right)$ is a Dirichlet process, by the definition of a Dirichlet process, we have

and therefore,

$$
P\left(\pi_{j k} \mid a, m\right)=\operatorname{Beta}\left(a m_{k}, a \sum_{\{k \prime \neq k\}} m_{k \prime}\right),
$$

$$
\mathrm{P}\left(\pi_{j k} \mid x_{\neg j i}, a, m\right)=\operatorname{Beta}\left(R_{j k}^{\neg j i}+a m_{k}, a \sum_{\{k \prime \neq k\}} R_{j k \prime}^{\neg j i}+m_{k \prime}\right)
$$

where $R_{j k}^{\neg i} \triangleq \sum_{i / \neq i}^{n_{j}} \mathbb{I}\left(x_{j i}=k\right)$. As such, the expected value of $\pi_{j k}$ is

$$
P\left(x_{j i}=k \mid x_{\neg j i}, a, m\right)=\frac{R_{j k}^{\neg j i}+a m_{k}}{R_{j .}^{\neg j i}+a},
$$

where $R_{j .}^{-j i}=\sum_{k=1}^{\infty} R_{j k}^{\neg i}$.

Given these likelihood and prior terms, the posterior is simply

$$
\begin{aligned}
P\left(x_{j i}=k \mid w_{j i}=v, x_{\neg j i}, w_{\neg j i}, b, \psi, a, m\right) & \propto \frac{S_{k v}^{\neg j i}+b \psi_{v}}{S_{k \cdot}^{\neg j i}+b} \frac{R_{j k}^{\neg j i}+a m_{k}}{R_{j}^{\neg j i}+a}, \\
& \propto \frac{S_{k v}^{\neg j i}+b \psi_{v}}{S_{k \cdot}^{\neg j i}+b} \times\left(R_{j k}^{\neg j i}+a m_{k}\right) .
\end{aligned}
$$

Note that from this, we also have

$$
\mathrm{P}\left(x_{j i}>\mathrm{K} \mid x_{\neg j i}, w, b, \psi, a, m\right) \propto \sum_{\{k>K\}} \frac{S_{k v}^{\neg j i}+b \psi_{v}}{S_{k \cdot}^{\neg j i}+b} \times\left(R_{j k}^{\neg j i}+a m_{k}\right) .
$$

Given that for all $k>K$, where $K$ is the maximum value of the set $\left\{x_{j i}: j \in\right.$ $\left.1 \ldots J, i \in 1 \ldots n_{j}\right\}$, we have $R_{j k}^{\neg j i}=0$ and $S_{k v}^{\neg j i}=0$, then

$$
\begin{aligned}
\mathrm{P}\left(x_{j i}>K \mid x_{-j i}, w, b, \psi, a, m\right) & \propto \sum_{\{k>K\}} \frac{b \psi_{v}}{b} \times a m_{k}, \\
& =\psi_{v} \times a m_{u}
\end{aligned}
$$

where $m_{\mathfrak{u}}=\sum_{\{k>k\}} m_{j k}$.

As a practical matter of sampling, for each latent variable $x_{j i}$, we calculate

$$
f_{j i k}=\propto \frac{S_{k v}^{\neg j i}+b \psi_{v}}{S_{k}^{\neg j i}+b} \times\left(R_{j k}^{\neg j i}+a m_{k}\right),
$$

for $k \in 1,2 \ldots K$, and then

$$
f_{j i u}=\psi_{v} \times a m_{u}
$$

where $K, m_{\mathfrak{u}}$ are defined as above and $v=w_{j i}$. Now,

$$
P\left(x_{j i} \leqslant K \mid x_{\neg j i}, w, b, \psi, a, m\right)=\frac{\sum_{k=1}^{K} f_{j i k}}{\sum_{k=1}^{K} f_{j i k}+f_{j i u}}
$$


and

$$
P\left(x_{j i}>K \mid x_{-j i}, w, b, \psi, a, m\right)=\frac{f_{j i u}}{\sum_{k=1}^{K} f_{j i k}+f_{j i u}},
$$

and so a single random sample will be sufficient to decide if $x_{j i} \leqslant K$ or $x_{j i}>K$. If $x_{j i} \leqslant K$, then

$$
P\left(x_{j i}=k \mid x_{\neg j i}, w, b, \psi, a, m, x_{j i} \leqslant K\right)=\frac{f_{j i k}}{\sum_{k=1}^{K} f_{j i k}} .
$$

On the other hand, if $x_{j i}>K$, the probability that $x_{j i}=k^{\text {new }}$ for $k^{\text {new }}>K$ is

$$
\mathrm{P}\left(x_{j i}=k^{\text {new }} \mid x_{\neg j i}, w, b, \psi, a, m, x_{j i}>K\right)=\frac{\psi_{v} \times a m_{k^{n e w}}}{f_{j i u}}=\frac{m_{k^{n e w}}}{m_{u}} .
$$

\section{Sampling $\mathrm{m}$ and $\mathrm{a}$}

The posterior distribution over the infinite length array $m$ is

$$
\mathrm{P}\left(\mathrm{m} \mid \mathrm{x}_{1: \mathrm{J}}, \mathrm{a}, \gamma\right) \propto \mathrm{P}\left(\mathrm{x}_{1: \mathrm{J}} \mid \mathrm{a}, \mathrm{m}\right) \mathrm{P}(\mathrm{m} \mid \gamma),
$$

while the posterior over the scalar parameter $a$ is

$$
P\left(a \mid x_{1: J}\right) \propto P\left(x_{1: J} \mid a, m\right) P(a),
$$

with the priors as stated above. The likelihood term in both cases is

$$
\mathrm{P}\left(x_{1: j} \mid \mathrm{a}, \mathrm{m}\right)=\prod_{j=1}^{J} \int \prod_{i=1}^{n_{j}} \mathrm{P}\left(x_{j i} \mid \pi_{j}\right) \mathrm{P}\left(\pi_{j} \mid a, m\right) \mathrm{d} \pi_{j},
$$

where $\prod_{i=1}^{n_{j}} P\left(x_{j i} \mid \pi_{j}\right)=\prod_{k=1}^{K} \pi_{j k}^{R_{j k}}$, with $R_{j k}=\sum_{i=1}^{n_{j}} \mathbb{I}\left(x_{j i}=k\right)$ and $K$ is, as stated above, the maximum value attained by any latent variable. The prior $\mathrm{P}\left(\pi_{\mathrm{j}} \mid \mathrm{a}, \mathrm{m}\right)$ is a Dirichlet process prior and so, by definition of the Dirichlet process,

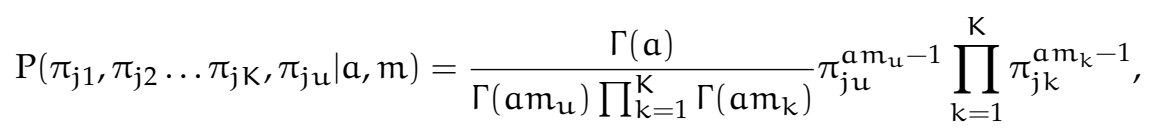

where $m_{\mathfrak{u}}=\sum_{\{k>k\}} m_{j k}$, as stated above, and $\pi_{\mathfrak{u}}=\sum_{\{k>k\}} \pi_{j k}$. Therefore,

$$
\begin{aligned}
& \mathrm{P}\left(x_{1: \mathrm{J}} \mid \mathrm{a}, \mathrm{m}\right)=\prod_{j=1}^{J} \int \prod_{i=1}^{n_{j}} \mathrm{P}\left(x_{j i} \mid \pi_{j}\right) \mathrm{P}\left(\pi_{j} \mid \mathrm{a}, \mathrm{m}\right) \mathrm{d} \pi_{j}, \\
& =\prod_{j=1}^{J} \frac{\Gamma(a)}{\Gamma\left(a m_{\mathfrak{u}}\right) \prod_{k=1}^{\mathrm{K}} \Gamma\left(\mathrm{a} m_{k}\right)} \pi_{j \mathfrak{u}}^{\mathrm{a} m_{\mathfrak{u}}-1} \int \prod_{k=1}^{\mathrm{K}} \pi_{j k}^{\mathrm{R}_{\mathrm{jk}}+\mathrm{m}_{\mathrm{k}}-1} \mathrm{~d} \pi_{\mathrm{j}}, \\
& =\prod_{j=1}^{J} \frac{\Gamma(a)}{\Gamma\left(a m_{\mathfrak{u}}\right) \prod_{k=1}^{K} \Gamma\left(a m_{k}\right)} \frac{\Gamma\left(a m_{\mathfrak{u}}\right) \prod_{k=1}^{K} \Gamma\left(R_{j k}+a m_{k}\right)}{\Gamma\left(R_{j}+a\right)}, \\
& =\prod_{j=1}^{J} \frac{\Gamma(a)}{\Gamma\left(R_{j}+a\right)} \prod_{k=1}^{K} \frac{\Gamma\left(R_{j k}+a m_{k}\right)}{\Gamma\left(a m_{k}\right)}
\end{aligned}
$$

and this can be re-written as

$$
=\prod_{j=1}^{J} \frac{1}{\Gamma\left(R_{j} .\right)} \int_{0}^{1}\left(\tau_{j}^{r}\right)^{a-1}\left(1-\tau_{j}^{r}\right)^{R_{j} .-1} d \tau_{j}^{r} \prod_{k=1}^{K} \sum_{\sigma_{j k}^{r}=0}^{R_{j k}} \mathbb{S}\left(R_{j k}, \sigma_{j k}^{r}\right)\left(a m_{k}\right)^{\sigma_{j k}^{r},}
$$


given that

$$
\int_{0}^{1}\left(\tau_{j}^{r}\right)^{a-1}\left(1-\tau_{j}^{r}\right)^{R_{j} .-1} d \tau_{j}^{r} \doteq \frac{\Gamma(a) \Gamma\left(R_{j} .\right)}{\Gamma\left(R_{j} .+a\right)}
$$

and

$$
\sum_{\sigma_{j k}^{r}=0}^{R_{j k}} \mathbb{S}\left(R_{j k}, \sigma_{j k}^{r}\right)\left(a m_{k}\right)^{\sigma_{j k}^{r}} \doteq \frac{\Gamma\left(R_{j k}+a m_{k}\right)}{\Gamma\left(a m_{k}\right)},
$$

where $\mathbb{S}$ is an unsigned Stirling number of the first kind. By treating $\tau^{r}$ and $\sigma^{r}$ as unobserved auxiliary variable, this leads to the augmented likelihood term

$$
\mathrm{P}\left(x_{1: J} \mid \mathrm{a}, \mathrm{m}, \tau^{r}, \sigma^{r}\right)=\prod_{j=1}^{J} \frac{1}{\Gamma\left(R_{j} .\right)}\left(\tau_{j}^{r}\right)^{a-1}\left(1-\tau_{j}^{r}\right)^{R_{j} \cdot-1} \prod_{k=1}^{K} \mathbb{S}\left(R_{j k}, \sigma_{j k}^{r}\right)\left(a m_{k}\right)^{\sigma_{j k}^{r}} .
$$

With the augmented likelihood treated as a function of $\sigma_{j k}^{r}$, we have

$$
P\left(x_{1: j} \mid a, m, \sigma^{r}\right) \propto \mathbb{S}\left(R_{j k}, \sigma_{j k}^{r}\right)\left(a m_{k}\right)^{\sigma_{j k}^{r}} .
$$

and with a uniform prior over the values of $\sigma_{j k}^{r}$, the posterior probability of $\sigma_{j k}^{r}$ given all other variables is therefore

$$
P\left(\sigma_{j k}^{r} \mid x_{1: J}, a, m\right)=\frac{\mathbb{S}\left(R_{j k}, \sigma_{j k}^{r}\right)\left(a m_{k}\right)^{\sigma_{j k}^{r}}}{\sum_{\sigma_{j k}^{r}=0}^{R_{j k}} \mathbb{S}\left(R_{j k}, \sigma_{j k}^{r}\right)\left(a m_{k}\right)^{\sigma_{j k}^{r}}} .
$$

On the other hand, when the augmented likelihood is treated as a function of $\tau_{j}^{r}$, we have

$$
P\left(x_{1: J} \mid a, \tau_{j}^{r}\right) \propto\left(\tau_{j}^{r}\right)^{a-1}\left(1-\tau_{j}^{r}\right)^{R_{j} \cdot-1},
$$

and so, with a uniform prior on $\tau_{j}^{r}$, the posterior is

$$
P\left(\tau_{j}^{r} \mid x_{1: J}, a\right) \propto\left(\tau_{j}^{r}\right)^{a-1}\left(1-\tau_{j}^{r}\right)^{R_{j} \cdot-1}=\operatorname{Beta}\left(a, R_{j} .\right) .
$$

Similarly, with the augmented likelihood treated as function of $m$, we have

$$
P\left(x_{1: J} \mid m, \sigma^{r}\right) \propto \prod_{k=1}^{K} m_{k}^{\sum_{j=1}^{J} \sigma_{j k}^{r}}=\prod_{k=1}^{K} m_{k}^{\sigma_{\cdot k}^{r}} .
$$

The prior on $m$ is a stick-breaking prior, and so the probability distribution over $m_{1} \ldots m_{k} \ldots m_{k}, m_{\mathfrak{u}}$ is $m_{1} \sim \omega_{1}^{0}$, and then $m_{k} \sim \omega_{k}^{0}\left(1-\sum_{k \prime=1}^{k-1} m_{k^{\prime}}\right)$ for $k \in 2 \ldots k$, and finally $m_{\mathfrak{u}}=1-\sum_{k=1}^{K} m_{k}$, where $\omega_{1}^{0} \ldots \omega_{K}^{0}$ are independently and identically distributed as $\operatorname{Beta}(1, \gamma)$. This finite stick-breaking distribution is a $\mathrm{K}+1$ dimensional Generalized Dirichlet distribution, see Connor and Mosimann (1969); Wong (1998), whose parameter vectors are a vector of $K 1^{\prime}$ 's and a vector of $K \gamma^{\prime}$ s. As described in Wong (1998), the Generalized Dirichlet distribution is a conjugate prior for a multinomial likelihood. As such, the posterior over $m_{1}, m_{2} \ldots m_{K}, m_{\mathfrak{u}}$ is a Generalized Dirichlet distribution with length $K$ parameter vectors $\alpha_{1}, \alpha_{2} \ldots \alpha_{K}$ and $\beta_{1}, \beta_{2} \ldots \beta_{K}$, where

$$
\alpha_{k}=1+\sigma_{\cdot k^{\prime}}^{r}, \quad \beta_{k}=\gamma+\sum_{k \prime=k+1}^{K} \sigma_{\cdot k \prime}^{r}, \quad \text { for } k \in 1,2 \ldots k .
$$

We can sample from this Generalized Dirichlet distribution by using a finite stickbreaking construction, as was used for the prior, i.e. for $m_{1} \sim \omega_{1}, m_{k} \sim \omega_{k}(1-$ 
$\left.\sum_{k \prime=1}^{k-1} m_{k \prime}\right)$ for $k \in 1 \ldots K$, and $m_{\mathfrak{u}}=1-\sum_{k=1}^{K} m_{k}$, where $\omega_{k} \sim \operatorname{Beta}\left(\alpha_{k}, \beta_{k}\right)$.

Finally, when treated as a function of $a$, the augmented likelihood is

$$
P\left(x_{1: j} \mid m, \sigma^{r}\right)=\prod_{j=1}^{J}\left(\tau_{j}^{r}\right)^{a} \prod_{k=1}^{K} a^{\sigma_{j k}^{r}}=e^{a \sum_{j=1}^{J} \log \tau_{j}^{r}} a^{\sum_{j k} \sigma_{j k}^{r}} .
$$

As stated above, the prior on a is a Gamma distribution whose shape and scale parameters are both equal to 1.0. Therefore, the posterior is a Gamma distribution with shape and scale

$$
\sum_{j k} \sigma_{j k}^{r}+1, \frac{1}{1-\sum_{j=1}^{J} \log \tau_{j}^{r}}
$$

Here, we assume the following parameterization of the Gamma distribution:

$P(x \mid a, s)=\frac{1}{s^{a} \Gamma(a)} x^{a-1} e^{-\frac{x}{s}}$,

where $\mathrm{a}$ and $\mathrm{s}$ are the shape and scale parameters, respectively.

respectively.

\section{Sampling $\psi$ and $b$}

The posterior distribution over $\psi$ is

$$
\mathrm{P}\left(\psi \mid w_{1: \mathrm{J}}, x_{1: \mathrm{J}}\right) \propto \mathrm{P}\left(w_{1: \mathrm{J}} \mid x_{1: \mathrm{J}}, \psi, \mathrm{b}\right) \mathrm{P}(\psi \mid \mathrm{c}),
$$

while the posterior over the concentration parameter $b$ is

$$
\mathrm{P}\left(\mathrm{b} \mid w_{1: \mathrm{J}}, \mathrm{x}_{1: \mathrm{J}}\right) \propto \mathrm{P}\left(w_{1: \mathrm{J}} \mid \mathrm{x}_{1: \mathrm{J}}, \psi, \mathrm{b}\right) \mathrm{P}(\mathrm{b}) .
$$

In both cases, the likelihood term is

$$
\begin{aligned}
& \mathrm{P}\left(w_{1: \mathrm{J}} \mid x_{1: \mathrm{J}}, \psi, \mathrm{b}\right)=\int \prod_{j=1}^{\mathrm{J}} \prod_{i=1}^{n_{j}} \mathrm{P}\left(w_{j i} \mid x_{j i}, \phi_{1}, \phi_{2} \ldots\right) \mathrm{P}\left(\phi_{1}, \phi_{2} \ldots \mid \psi, \mathrm{b}\right) \mathrm{d} \phi_{1} \mathrm{~d} \phi_{2} \ldots, \\
& =\int \prod_{j=1}^{J} \prod_{i=1}^{n_{j}} P\left(w_{j i} \mid \phi_{x_{j i}}\right) \prod_{k=1}^{\infty} P\left(\phi_{k} \mid \psi, b\right) d \phi_{1} d \phi_{2} \ldots, \\
& =\underbrace{\prod_{k=K+1}^{\infty} \int P\left(\phi_{k} \mid \psi, b\right) d \phi_{k}}_{=1} \int \prod_{j=1}^{J} \prod_{i=1}^{n_{j}} \mathrm{P}\left(w_{j i} \mid \phi_{x_{j i}}\right) \prod_{k}^{K} P\left(\phi_{k} \mid \psi, b\right) d \phi_{1} d \phi_{2} \ldots, \\
& =\frac{\Gamma(\mathrm{b})}{\prod_{v=1}^{V} \Gamma\left(\mathrm{b} \psi_{v}\right)} \prod_{\mathrm{k}=1}^{\mathrm{K}} \int \prod_{v=1}^{\mathrm{V}} \phi_{\mathrm{kv}}^{\mathrm{S}_{\mathrm{k} v}-\mathrm{b} \psi_{v}-1} \mathrm{~d} \phi_{\mathrm{k}}, \\
& =\prod_{\mathrm{k}=1}^{\mathrm{K}} \frac{\Gamma(\mathrm{b})}{\Gamma\left(\mathrm{S}_{\mathrm{k} \cdot}+\mathrm{b}\right)} \prod_{v=1}^{\mathrm{V}} \frac{\Gamma\left(\mathrm{S}_{\mathrm{k} v}+\mathrm{b} \psi_{v}\right)}{\Gamma\left(\mathrm{b} \psi_{v}\right)},
\end{aligned}
$$

and, as was the case for $\mathrm{P}\left(\mathrm{x}_{1: \mathrm{J}} \mid \mathrm{a}, \mathrm{m}\right)$, this likelihood can be rewritten as

$\mathrm{P}\left(w_{1: J} \mid x_{1: J}, \psi, b\right)=\prod_{k=1}^{\mathrm{K}} \frac{1}{\Gamma\left(S_{k}\right)} \int_{0}^{1}\left(\tau_{k}^{s}\right)^{b-1}\left(1-\tau_{k}^{s}\right)^{S_{k} \cdot-1} \mathrm{~d} \tau_{k}^{s} \prod_{v=1}^{V} \sum_{\sigma_{k v}^{s}=0}^{S_{k v}} \mathbb{S}\left(S_{k v}, \sigma_{k v}^{s}\right)\left(b \psi_{v}\right)^{\sigma_{k v}^{s}}$.

and by treating $\sigma^{s}$ and $\tau^{s}$ as auxiliary variables, we obtain the augmented likelihood

$$
\begin{aligned}
& \mathrm{P}\left(w_{1: \mathrm{J}} \mid \mathrm{x}_{1: \mathrm{J},}, \sigma^{\mathrm{s}}, \tau^{\mathrm{s}}, \psi, \mathrm{b}\right) \\
& =\prod_{k=1}^{\mathrm{K}} \frac{1}{\Gamma\left(S_{\mathrm{k} \cdot}\right)}\left(\tau_{\mathrm{k}}^{\mathrm{s}}\right)^{\mathrm{b}-1}\left(1-\tau_{\mathrm{k}}^{\mathrm{s}}\right)^{S_{\mathrm{k} \cdot}-1} \prod_{v=1}^{\mathrm{V}} \mathbb{S}\left(S_{\mathrm{k} v}, \sigma_{\mathrm{k} v}^{\mathrm{s}}\right)\left(\mathrm{b} \psi_{v}\right)^{\sigma_{\mathrm{k} v}^{\mathrm{s}} .} .
\end{aligned}
$$


As the prior for $\psi$ is a symmetric Dirichlet with concentration parameter c, its posterior is the Dirichlet distribution

$$
\mathrm{P}\left(\psi \mid \sigma^{\mathrm{s}}, \mathrm{c}, \mathrm{V}\right)=\propto \prod_{\nu=1}^{\mathrm{V}} \psi_{\nu} \sum_{\mathrm{k}} \sigma_{\mathrm{k} v}^{\mathrm{s}}+\mathrm{c} / \mathrm{V}-1 .
$$

On the other hand, just like with the case of $\sigma_{j k}^{r}$, the posterior for each $\sigma_{k \nu}^{s}$ is

$$
\mathrm{P}\left(\sigma_{k v}^{s} \mid w, x, \psi, b\right)=\frac{\mathbb{S}\left(S_{k v}, \sigma_{k v}^{s}\right) b \psi_{\nu}^{\sigma_{k v}^{s}}}{\sum_{\sigma_{k v}^{s}=0}^{S_{k v}} \mathbb{S}\left(S_{k v}, \sigma_{k v \prime}^{s}\right) b \psi_{v}^{\sigma_{k \nu \prime}^{s}}}
$$

For the case of $b$, its prior is a Gamma distribution with shape and scale parameters equal to 1 . The augmented likelihood treated as a function of $b$ is

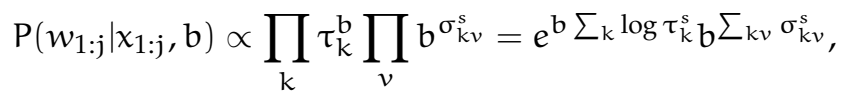

and so the posterior is a Gamma distribution with shape and scale

$$
\sum_{k v} \sigma_{k v}^{s}+1, \frac{1}{1-\sum_{k} \log \tau_{k}^{s}}
$$

respectively. Finally, with a uniform prior on each $\tau_{k}^{s}$, and given that the augmented treated as a function of $\tau_{\mathrm{k}}^{s}$ is

$$
\mathrm{P}\left(w_{1: \mathrm{J}} \mid x_{1: J}, \mathrm{~b}, \tau_{\mathrm{j}}^{\mathrm{r}}\right)=\left(\tau_{\mathrm{k}}^{\mathrm{s}}\right)^{\mathrm{b}-1}\left(1-\tau_{\mathrm{k}}^{\mathrm{s}}\right)^{S_{\mathrm{k} \cdot}-1}
$$

the posterior for each $\tau_{k}^{s}$ is $\operatorname{Beta}\left(b, S_{k}\right.$. $)$.

\section{Sampling $c$}

Rather than inferring $c$, the hyperprior of $\psi$, directly from the sampled value of $\psi$, we can infer $c$ on the basis of the remaining observed variables, integrating over $\phi$ and $\psi$. The probability of the observed variables, integrating over $\phi$ and $\psi$, is

$$
\mathrm{P}\left(w_{1: \mathrm{J}} \mid x_{1: \mathrm{J}}, \mathrm{b}, \mathrm{c}\right)=\int \underbrace{\int \mathrm{P}\left(w_{1: \mathrm{J}} \mid x_{1: \mathrm{J}}, \phi\right) \mathrm{P}(\phi \mid \mathrm{b}, \psi) \mathrm{d} \phi}_{\mathrm{P}\left(w_{1: \mathrm{J}} \mid x_{1: \mathrm{J},}, \psi, \mathrm{b}\right)} \mathrm{P}(\psi \mid \mathbf{c}) \mathrm{d} \psi .
$$

Using the augmented version of $\mathrm{P}\left(w_{1: J} \mid x_{1: J}, \psi, b\right)$ from the previous section, i.e., $\mathrm{P}\left(w_{1: \mathrm{J}} \mid x_{1: \mathrm{J}}, \sigma^{s}, \tau^{s}, \psi, \mathrm{b}\right)$, and treating this augmented likelihood as a function of $\psi$, we then have

$$
\begin{aligned}
\int \mathrm{P}\left(w_{1: \mathrm{J}} \mid \sigma^{\mathrm{s}}, \psi\right) \mathrm{P}(\psi \mid \mathrm{c}) \mathrm{d} \psi & \propto \int \prod_{v=1}^{\mathrm{V}} \psi_{v}^{\sum_{k=1}^{\mathrm{K}} \sigma_{\mathrm{k} v}^{\mathrm{s}}} \prod_{v=1}^{\mathrm{V}} \psi_{v}^{\mathrm{c} / \mathrm{V}-1} \mathrm{~d} \psi, \\
& =\frac{\Gamma(\mathrm{c})}{\Gamma\left(\mathrm{c}+\sigma_{. .}^{\mathrm{s}}\right)} \prod_{v=1}^{\mathrm{V}} \frac{\Gamma\left(\sigma_{\cdot v}^{\mathrm{s}}+\mathrm{c} / \mathrm{V}\right)}{\Gamma(\mathrm{c} / \mathrm{V})},
\end{aligned}
$$

which is the likelihood function for c. As before, we can re-write this likelihood as

$$
\frac{1}{\Gamma\left(\sigma_{. .}^{s}\right)} \int\left(\tau^{q}\right)^{\mathfrak{c}-1}\left(1-\tau^{q}\right)^{\sigma_{. .}^{s}-1} \mathrm{~d} \tau^{q} \prod_{v=1}^{V} \sum_{\sigma_{v}^{q}=0}^{\sigma_{v}^{s}} \mathbb{S}\left(\sigma_{. v}^{s}, \sigma_{v}^{q}\right)\left(\frac{c}{V}\right)^{\sigma_{v}^{q}}
$$


and treating $\tau^{q}$ and $\sigma^{q}$ as auxiliary variables, we have the augmented likelihood

$$
\frac{1}{\Gamma\left(\sigma_{. .}^{s}\right)}\left(\tau^{q}\right)^{c-1}\left(1-\tau^{q}\right)^{\sigma_{. .}^{s}-1} \prod_{v=1}^{V} \mathbb{S}\left(\sigma_{\cdot v}^{s}, \sigma_{v}^{q}\right)\left(\frac{c}{V}\right)^{\sigma_{v}^{q}} .
$$

Following the same procedure here as we used for the case of $a$ and $m$, and $b$ and $\psi$, the posterior for each $\sigma_{v}^{q}$ is

$$
\frac{\mathbb{S}\left(\sigma_{\cdot v^{\prime}}^{\mathrm{s}}, \sigma_{v}^{\mathrm{q}}\right) \frac{\mathrm{c}}{V} \sigma_{v}^{\mathrm{q}}}{\sum_{\sigma_{v \prime}^{\mathrm{q}}=0}^{\sigma_{v}} \mathbb{S}\left(\sigma_{\cdot v^{\prime}}^{s}, \sigma_{v \prime}^{\mathrm{q}}\right) \frac{\mathrm{c}}{V} \sigma_{v \prime}^{\mathrm{q}}} .
$$

The posterior for $\mathrm{c}$ is the Gamma distribution with shape and scale

$$
\sum_{v=1}^{V} \sigma_{v}^{q}+1, \frac{1}{1-\log \tau^{q}}
$$

respectively, and the posterior for $\tau^{q}$ is $\operatorname{Beta}\left(c, \sigma_{\text {.. }}^{\text {s. }}\right)$.

\section{Sampling $\gamma$}

The posterior distribution distribution for the stick-breaking parameter $\gamma$ is

$$
\mathrm{P}(\gamma \mid \mathrm{m}) \propto \mathrm{P}(\mathrm{m} \mid \gamma) \mathrm{P}(\gamma) .
$$

As $m_{1}, m_{2} \ldots m_{K}, m_{\mathfrak{u}}$ is a deterministic function of $\omega_{1}, \omega_{2} \ldots \omega_{K}$, the likelihood of $m$ given $\gamma$ is equivalent to the likelihood of $\omega$ given $\gamma$, which is a product of Beta distributions

$$
\begin{aligned}
P(\omega \mid \gamma) & =\prod_{k=1}^{\mathrm{K}} \operatorname{Beta}\left(\omega_{k} \mid 1, \gamma\right) \\
& \propto \frac{\Gamma(1+\gamma)^{\mathrm{K}}}{\Gamma(\gamma)} \prod_{k=1}^{\mathrm{K}}\left(1-\omega_{\mathrm{k}}\right)^{\gamma-1}, \\
& =\gamma^{\mathrm{K}} e^{\gamma \sum_{k} \log \left(1-\omega_{k}\right)}
\end{aligned}
$$

For any $a, \frac{\Gamma(1+a)}{\Gamma(a)}=a$

With a Gamma prior on $\gamma$, whose shape and scale equal 1, the posterior is also a Gamma distribution with shape and scale

$$
\mathrm{K}+1, \quad \frac{1}{1-\sum_{\mathrm{k}} \log \left(1-\omega_{\mathrm{k}}\right)} .
$$

respectively.

\section{References}

Blei, D., Ng, A., \& Jordan, M. (2003). Latent dirichlet allocation. Journal of Machine Learning Research, 3, 993-1022.

Connor, R. J., \& Mosimann, J. E. (1969). Concepts of independence for proportions with a generalization of the dirichlet distribution. Journal of the American Statistical Association, 64(325), 194-206.

Newman, D., Asuncion, A., Smyth, P., \& Welling, M. (2009). Distributed algorithms for topic models. The Journal of Machine Learning Research, 10, 1801-1828. 
Teh, Y. W., Jordan, M. I., Beal, M. J., \& Blei, D. M. (2004). Hierarchical Dirichlet Processes. In Advances in neural information processing systems (Vol. 17).

Teh, Y. W., Jordan, M. I., Beal, M. J., \& Blei, D. M. (2006, DEC). Hierarchical Dirichlet processes. Journal of the American Statistical Association, 101(476), 1566-1581.

Wong, T.-T. (1998). Generalized dirichlet distribution in bayesian analysis. Applied Mathematics and Computation, 97(2), 165-181. 